Cite this: Soft Matter, 2013, 9, 10025

\title{
Mechanistic control over morphology: self-assembly of a discotic amphiphile in water
}

\author{
Isja de Feijter, ${ }^{\text {abc }}$ Pol Besenius, ${ }^{d}$ Lorenzo Albertazzi, $^{\text {ac }}$ E. W. Meijer, ${ }^{\text {abc }}$ \\ Anja R. A. Palmans ${ }^{\mathrm{ab}}$ and Ilja K. Voets ${ }^{\star a b}$
}

We report on the self-assembly of discotic amphiphiles that contain chelated gadolinium(III) ions and are based on the $C_{3}$-symmetrical benzene-1,3,5-tricarboxamide motif. Fluorescence spectroscopy, SAXS and cryo-TEM experiments demonstrate that a bimodal distribution of small and large aggregates is formed

Received 5th August 2013

Accepted 21st August 2013

DOI: $10.1039 / \mathrm{c} 3 \mathrm{sm} 52104 d$

in a ratio that is dependent on the ionic strength. The results correlate with the previously reported degree of cooperativity of the polymerization mechanism, which increases with increasing $\mathrm{NaCl}$ concentration. Hence, by tuning the electrostatic interactions between the ligands at the periphery we can tune the cooperativity of the self-assembly. This tunability provides a versatile handle to adjust the

www.rsc.org/softmatter size and shape of the discotic amphiphiles, which have potential as supramolecular MRI contrast agents.

\section{Introduction}

In the past, a wide range of supramolecular self-assembling motifs have been extensively studied in organic solvents. ${ }^{\mathbf{1 - 4}}$ More recently, there has been increasing interest in the transformation of these motifs to facilitate self-assembly in aqueous media. A detailed understanding of the self-assembly mechanism and morphology of these modified motifs is of major importance as it renders them applicable for biologically relevant applications. Recent examples include their use as hydrogels, ${ }^{5-8}$ cellular uptake carriers ${ }^{9}$ and imaging agents. ${ }^{10,11}$ The translation from self-assembly in organic solvents into aqueous media is, however, far from trivial. ${ }^{12}$ To create structures that are water compatible, a multitude of strategies has been applied: attachment of oligo(ethylene oxide) side chains, ${ }^{\mathbf{1 3 - 1 5}}$ sugar moieties ${ }^{\mathbf{1 6 - 1 8}}$ or other hydrophilic groups. ${ }^{19}$ We selected a triply charged gadolinium(III) chelate to induce water compatibility, with use as a magnetic resonance imaging (MRI) contrast agent in mind.

Self-assembling discotic amphiphiles based on the $C_{3^{-}}$ symmetrical benzene-1,3,5-tricarboxamide (BTA) motif that contain gadolinium(III) ions chelated by diethylene triamine pentaacetic acid (DTPA) (from now on referred to as Gd(III)DTPA-BTA) (Fig. 1) have been shown to significantly enhance

${ }^{a}$ Institute for Complex Molecular Systems, Eindhoven University of Technology, P.O. Box 513, 5600 MB Eindhoven, The Netherlands

${ }^{b}$ Laboratory of Macromolecular and Organic Chemistry, Department of Chemical Engineering and Chemistry, Eindhoven University of Technology, The Netherlands. E-mail: i.voets@tue.nl; Tel: +31402473101

${ }^{c}$ Laboratory of Chemical Biology, Department of Biomedical Engineering, Eindhoven University of Technology, The Netherlands

${ }^{d}$ Organic Chemistry Institute \& CeNTech, Westfälische Wilhelms-Universität Münster, Corrensstrasse 40, 48149 Münster, Germany the contrast in magnetic resonance angiography of mouse brains. ${ }^{10}$ The non-covalent interactions between the selfassembling units on the one hand allow the formation of high molecular weight supramolecular polymers which enhance MRI contrast, and on the other hand provide reversibility and easy excretion of the aggregates.

The properties and self-assembly characteristics of BTAs in apolar solvents are well established in the literature, ${ }^{\mathbf{3 , 2 0}}$ and more recently the self-assembly of various BTAs in water has also been studied. ${ }^{21-24} \mathrm{Gd}(\mathrm{III})$-DTPA-BTA self-assembly in water occurs via a frustrated growth mechanism which results in a markedly different morphology compared to BTA self-assembly in apolar solvents. ${ }^{25}$ Instead of long rod-like structures, welldefined particles of approximately $6 \mathrm{~nm}$ are obtained. ${ }^{25}$ The frustrated growth is a result of the balance between attractive hydrophobic interactions and hydrogen bonding between the BTA amides, and repulsive interactions between the negatively charged chelates at the periphery of the molecule. The influence of screening of the charges with $\mathrm{NaCl}$ on the cooperativity of this system was evaluated by CD-spectroscopy as a function of temperature and ionic strength. The cooperativity was found to

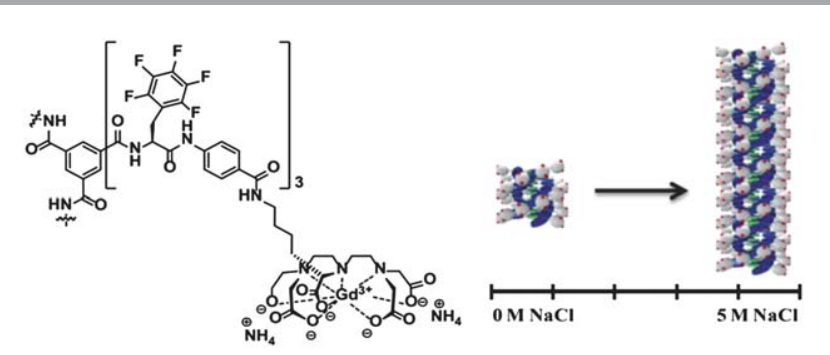

Fig. 1 Pentafluoro phenylalanine extended benzene-1,3,5-tricarboxamide that contains gadolinium(III) ions chelated by diethylene triamine pentaacetic acid and a schematic representation of the influence of $\mathrm{NaCl}$ on the self-assembly. 
increase from a nearly isodesmic polymerization to a cooperative growth mechanism. ${ }^{26}$ Furthermore, long rods were observed at high ionic strength $\left(c_{\mathrm{NaCl}}=5 \mathrm{M}\right){ }^{25}$

In this article we describe how changes in these external conditions result in the coexistence of small oligomers and elongated supramolecular polymers of Gd(III)-DTPA-BTA in the cooperative polymerization regime. While the coexistence of spherical and rod-like aggregates was previously observed in a number of surfactant and block copolymer amphiphilic systems, ${ }^{27-30}$ here, the coexistence of different types of helical supramolecular aggregates induced by a change in the polymerization mechanism is observed. The morphology of the supramolecular aggregates was studied using complementary techniques: cryogenic-transmission electron microscopy (cryoTEM), small-angle X-ray scattering (SAXS), and fluorescence spectroscopy using Nile Red as a fluorescent probe.

\section{Experimental section}

\section{Synthesis of Gd(III)-DTPA-BTA}

Gd(III)-DTPA-BTA was synthesized as described previously by Besenius et al. ${ }^{25}$ by coupling a hydrophobic spacer composed of perfluoro-t-phenylalanine and aminobenzoate to benzene-1,3,5tricarboxylic acid chloride. Subsequent coupling of lysine functionalized DTPA and complexation of gadolinium ions yields the desired discotic amphiphile. The product was lyophilized after extensive dialysis. ESI-MS confirmed the formation of the desired product, and the $\mathrm{Gd}(\mathrm{III})$ content was determined with ICP-AES to be $13.8 \mathrm{wt} \%$ (theoretically $14.8 \mathrm{wt} \%$ ).

\section{Nile Red}

Photoluminescence spectra were recorded on a Varian Cary Eclipse Fluorescence Spectrometer. Gd(III)-DTPA-BTA was dissolved in $100 \mathrm{mM}$ citrate buffer $(\mathrm{pH}=6)$ to obtain a BTA concentration of $5 \mu \mathrm{M}$. The $\mathrm{NaCl}$ concentration of the buffer was varied between 0 and $5 \mathrm{M} \mathrm{NaCl}$ in $0.5 \mathrm{M}$ steps. $2 \mu \mathrm{l}$ Nile Red (NR) stock solution in methanol was added to obtain a final concentration of $5 \mu \mathrm{M}$. The samples were excited at $550 \mathrm{~nm}$ and emission spectra were collected from 580 to $700 \mathrm{~nm}$.

\section{Small-angle X-ray scattering}

The small-angle X-ray scattering (SAXS) measurements were performed at the Dutch-Belgian BM26B beamline at the ESRF in Grenoble (France). Gd(III)-DTPA-BTA was dissolved in $100 \mathrm{mM}$ citrate buffer $(\mathrm{pH}=6)$ with $c_{\mathrm{NaCl}}=0,0.05,0.1,0.2,0.4,0.75,1.0$, 1.5 or $3.0 \mathrm{M}$ to obtain a BTA concentration of $1 \mathrm{mM}$. A sampleto-detector distance of $6.23 \mathrm{~m}$ was used together with an X-ray photon wavelength of $1.24 \AA$. The observed $q$ range was $7 \times$ $10^{-3} \AA^{-1} \leq q \leq 0.12 \AA^{-1}$, where $q$ is the magnitude of the scattering vector $q=(4 \pi / \lambda) \sin (\theta / 2)$, and where $\lambda$ is the X-ray wavelength and $\theta$ is the scattering angle. SAXS images were recorded on a $2 \mathrm{D}$ multiwire gas filled detector with $512 \times 512$ pixels of $260 \mu \mathrm{m}^{2}$ in size. The $2 \mathrm{D}$ images were radially averaged to obtain the intensity $I(q) v s . q$ profiles. Standard data reduction procedures, i.e. subtraction of the empty capillary and buffer contribution and correction for the sample absorption, were applied. All scattering patterns were fitted simultaneously with SASfit software using either a form factor for homogeneous spheres, or cylinders, or a linear combination of both. The radii $(R)$ of the spheres and the cross-sectional radii $\left(R_{\mathrm{cs}}\right)$ of the rods were set as global parameters while the length $(L)$ and scattering contrast $(\eta)$ were allowed to vary per curve.

\section{Cryo-TEM}

CryoTEM experiments were performed on Gd(III)-DTPA-BTA samples dissolved in $100 \mathrm{mM}$ citrate buffer $(\mathrm{pH}=6)$ with $c_{\mathrm{NaCl}}=0,0.2,0.75,1.5,3.0$ or $5.0 \mathrm{M}$ to obtain a BTA concentration of $1 \mathrm{mM}$. The experiments were performed on the TU/e CryoTitan (FEI), using a Gatan cryo-holder operating at $-170^{\circ} \mathrm{C}$. The TU/e CryoTitan is equipped with a field emission gun (FEG) operating at $300 \mathrm{kV}$. Images were recorded using a $2 k \times 2 k$ Gatan CCD camera equipped with a post column Gatan Energy Filter (GIF). The sample vitrification procedure was carried out using an automated vitrification robot (FEI Vitrobot ${ }^{\mathrm{TM}}$ Mark III). CryoTEM grids, R2/2 Quantifoil Jena grids, were purchased from Quantifoil Micro Tools GmbH. Prior to the vitrification procedure the grids were surface plasma treated using a Cressington 208 carbon coater operating at $5 \mathrm{~mA}$ for $40 \mathrm{~s}$.

\section{Results}

\section{Influence of the ionic strength on Gd(III)-DTPA-BTA aggregates}

Two markedly different morphologies were observed for supramolecular helical aggregates consisting of Gd(III)-DTPABTAs. At low ionic strength $6 \mathrm{~nm}$ sized particles are formed whereas at $5 \mathrm{M} \mathrm{NaCl}$ long rods are obtained..$^{25}$ To investigate how the transition proceeds, we added the solvatochromic dye Nile Red (NR) to Gd(III)-DTPA-BTA solutions in citrate buffer (pH $=6$ ) with salt concentrations between 0 and $5 \mathrm{M} \mathrm{NaCl}$. NR is a fluorescent probe which is often used in biological systems $\mathrm{s}^{31-34}$ and is also applied successfully to detect morphological transitions in supramolecular aggregates..$^{35}$

In citrate buffer, the emission $\left(\lambda_{\text {max }, \mathrm{em}}\right)$ of $\mathrm{NR}$ is $654 \mathrm{~nm}$, with a very low fluorescence intensity, irrespective of the salt concentration. Upon addition of $5 \mu \mathrm{M}$ BTA, $\lambda_{\text {max,em }}$ remains approximately at $654 \mathrm{~nm}$, but the fluorescence intensity increases, due to incorporation of the dye into an apolar microenvironment. ${ }^{36}$ Increasing the BTA concentration to $1 \mathrm{mM}$ causes a further increase of the fluorescence intensity as shown for $c_{\mathrm{NaCl}}=5 \mathrm{M}$ in Fig. 2A. In contrast, when the ionic strength of the buffered solution is increased, a shift of $\lambda_{\text {max,em }}$ to $639 \mathrm{~nm}$ is observed (Fig. 2B and C). This hypsochromic shift is indicative of a reduced polarity of the microenvironment due to screening of the charged ligands. Interestingly, the peak width also increased, from $57 \mathrm{~nm}$ at $0 \mathrm{M} \mathrm{NaCl}$ to $87 \mathrm{~nm}$ at $5 \mathrm{M} \mathrm{NaCl}$ (Fig. 2D). These results are consistent with the incorporation of NR into the hydrophobic environment created by Gd(III)-DTPABTA aggregates. Increasing the ionic strength further reduces the apparent polarity of the microenvironment of the dye. The broadening of the emission peak suggests that multiple species coexist in solution, as narrow peaks are expected when only a single species is present. ${ }^{36,37}$ 

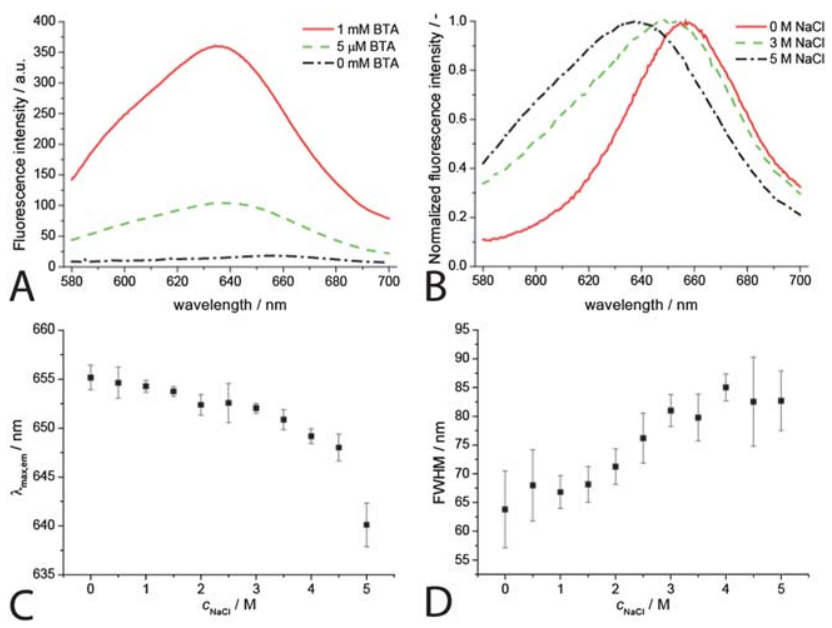

Fig. 2 Fluorescence spectroscopy of Gd(III)-DTPA-BTA in citrate buffer with an ionic strength between 0 and $5 \mathrm{M} \mathrm{NaCl}$ in the presence of solvatochromic dye Nile Red (measured in triplicate). (A) Increasing the Gd(III)-DTPA-BTA concentration at a fixed $c_{\mathrm{NaCl}}$ of $5 \mathrm{M}$ shows an increase of the fluorescence intensity; (B) increasing the $\mathrm{NaCl}$ concentration at a fixed $\mathrm{Gd}(\mathrm{III})$-DTPA-BTA concentration causes a blue shift of $\lambda_{\text {max,em }}$ as also shown in (C) and an increase of the peak width at half maximum (FWHM) as shown in (D).

\section{Coexistence of two distinctive morphologies}

SAXS experiments were performed to elucidate the size and shape of the supramolecular helical aggregates formed by Gd(III)-DTPA-BTAs as a function of the ionic strength. SAXS patterns were collected for $1 \mathrm{mM} \mathrm{Gd(III)-DTPA-BTA} \mathrm{samples} \mathrm{with}$ $\mathrm{NaCl}$ concentrations ranging from 0 to $3 \mathrm{M}$ (Fig. 3).

Three structural regimes can be distinguished on the basis of the obtained SAXS profiles. In the first regime, ranging from $0 \mathrm{M} \leq c_{\mathrm{NaCl}} \leq 0.1 \mathrm{M}$, scattering profiles are characteristic of objects with a low aspect ratio. A power law regime of $I \propto q^{-0}$ at low $q$-values is followed by a $I \propto q^{-4}$ regime at high $q$-values. Fitting the data to a form factor derived for homogeneous spheres yields a radius of $\pm 2.9 \mathrm{~nm}$, which is in excellent

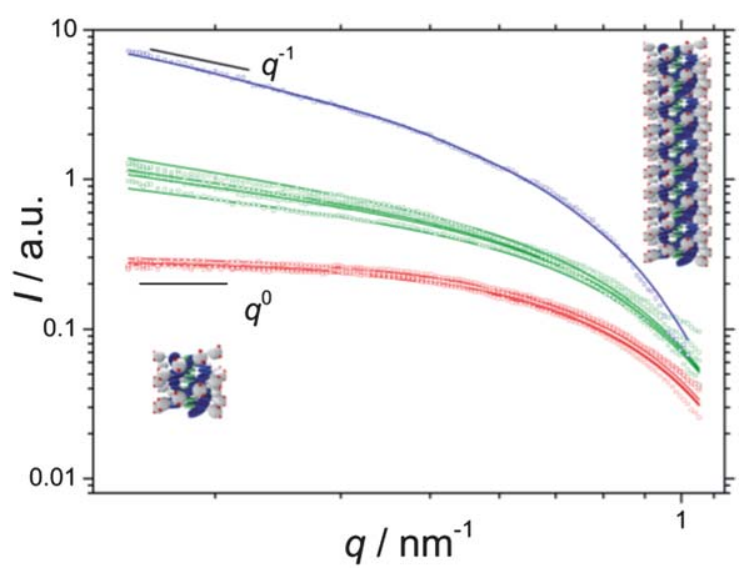

Fig. 3 SAXS patterns for $1 \mathrm{mM}$ BTA with $c_{\mathrm{NaCl}}$ ranging from 0 to $3 \mathrm{M}$ (symbols) and best fits to the data (continuous lines). Regime 1: $0-0.1 \mathrm{M} \mathrm{NaCl}$ in red, regime 2: $0.2-1.5 \mathrm{M} \mathrm{NaCl}$ in green and regime $3: 3 \mathrm{M} \mathrm{NaCl}$ in blue (curves shifted vertically by multiplication with 2 (green) and 7 (blue)). agreement with the calculated radius of the monomers. ${ }^{25}$ By contrast, at high salt concentrations $\left(c_{\mathrm{NaCl}}=3 \mathrm{M}\right)$, the profiles exhibit a regime with $I \propto q^{-1}$ at low $q$ which falls off to $I \propto q^{-4}$ at high $q$ values. This is a clear hallmark of rod-like objects with a length $L$ that is beyond the experimental resolution. The experimental data are well described by a form factor for rigid, homogeneous cylinders, which yields a cross-sectional radius of $\pm 2.9 \mathrm{~nm}$, again in good agreement with the molecular dimensions of $\mathrm{Gd}(\mathrm{III})-\mathrm{DTPA}-\mathrm{BTA}$. In the intermediate regime, where $0.2 \leq c_{\mathrm{NaCl}} \leq 1.5 \mathrm{M}$, neither of the form factors describes the data well. Instead, a linear combination of the two form factors was found to accurately capture the features of the scattering patters, such as the increase in forward scattering upon an increase in $c_{\mathrm{NaCl}}$. We find that the relative contribution of spherical particles to the total scattering intensity $(q \rightarrow 0)$ decreases linearly with increasing $c_{\mathrm{NaCl}}$, while vice versa, the relative contribution of cylindrical particles increases linearly (Fig. 4). We therefore conclude that an increasing number of monomers are incorporated into rods at the cost of monomers present in small oligomeric aggregates as $c_{\mathrm{NaCl}}$ increases.

Cryogenic transmission electron microscopy (cryo-TEM) experiments were carried out to corroborate the existence of three structural regimes in the salt induced transformation of small oligomers into long Gd(III)-DTPA-BTA aggregates (Fig. 5). Exclusively oligomers are observed at $0 \mathrm{M} \leq c_{\mathrm{NaCl}} \leq 0.2 \mathrm{M}$. A close examination of the images of $0.75 \mathrm{M}$ and $1.5 \mathrm{M} \mathrm{NaCl}$ reveals the presence of slightly elongated structures coexisting with the spherical particles. A bimodal distribution of small spherical aggregates and approximately $50 \mathrm{~nm}$ long rod-like structures is obtained at $3 \mathrm{M} \mathrm{NaCl}$. An increase of $c_{\mathrm{NaCl}}$ to $5 \mathrm{M}$ results in the formation of rods of approximately $200 \mathrm{~nm}$ in length. ${ }^{\mathbf{1 6}}$ At this $\mathrm{NaCl}$ concentration, spherical aggregates are not observed in the electron micrographs. Taking into account that the scattering of polydisperse mixtures is strongly dominated by the larger particles, we conclude that the SAXS and cryo-TEM give concordant results. In summary, Gd(III)-DTPABTAs self-assemble into exclusively globular objects at low $c_{\mathrm{NaCl}}$ and into cylindrical objects at high $c_{\mathrm{NaCl}}$. A bimodal distribution of oligomers and long aggregates is observed in the intermediate regime.

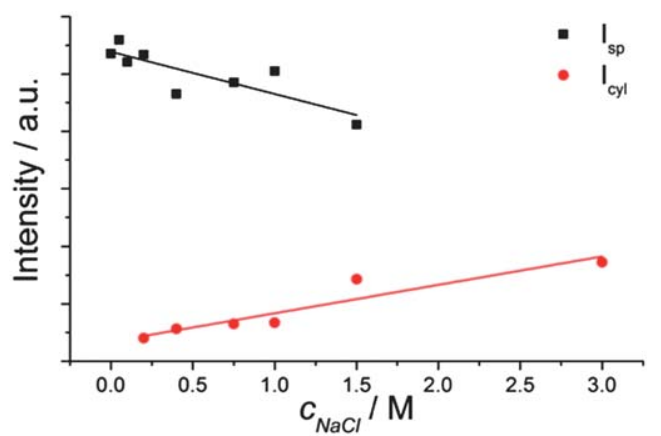

Fig. 4 Relative contribution of spherical (squares) and rod-like particles (dots) to the total scattering intensity, $I,(q \rightarrow 0)$ in a.u. 

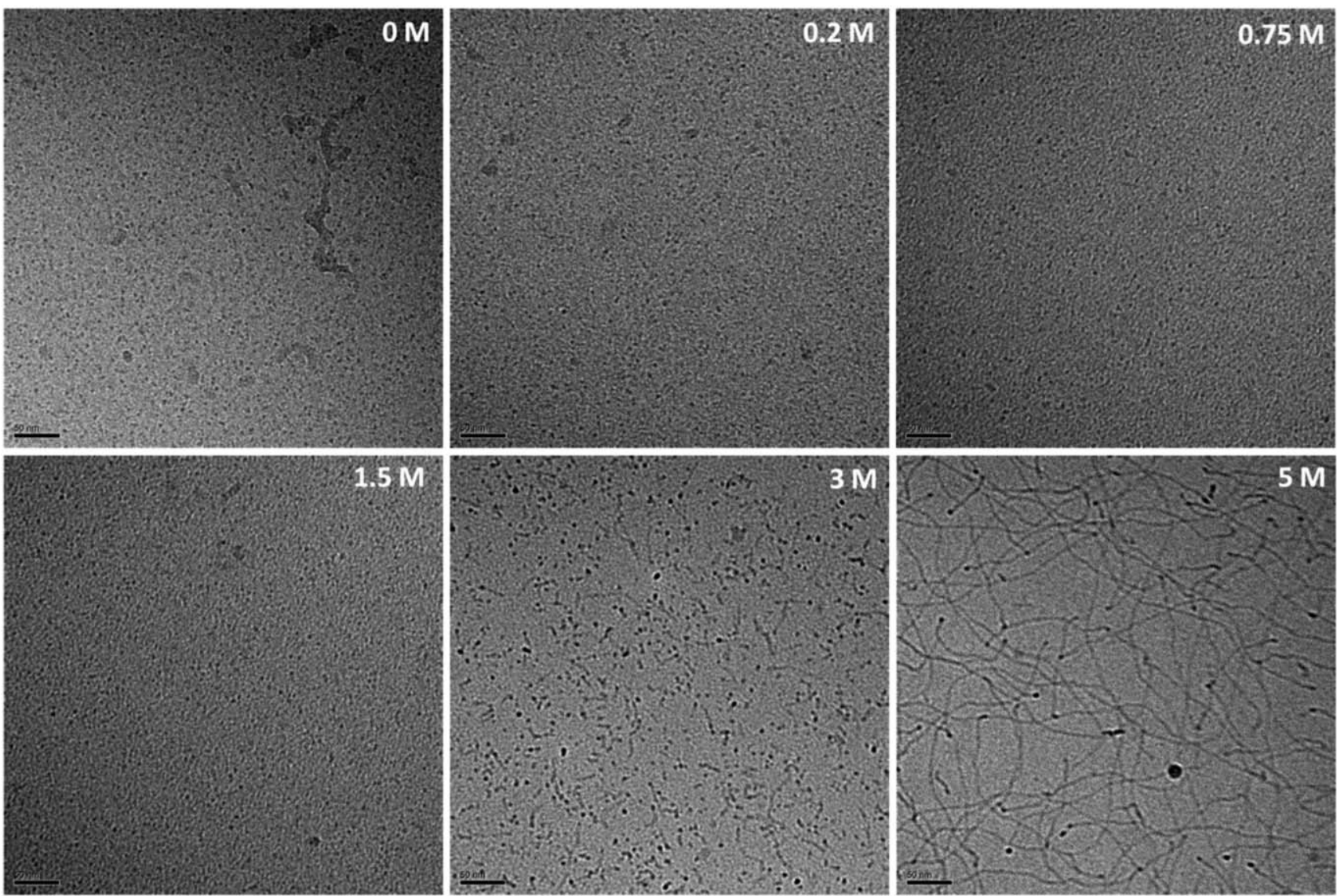

Fig. 5 Cryo-TEM images of aggregates composed of $1 \mathrm{mM} \mathrm{Gd(II)-DTPA-BTA}$ with 0, 0.2, 0,75, 1.5, 3 and $5 \mathrm{M} \mathrm{NaCl}$ in $100 \mathrm{mM}$ citrate buffer (pH =6); scale bar represents $50 \mathrm{~nm}$

\section{Discussion}

Recently, the cooperativity $\left(K_{\mathrm{a}}\right)$ of the supramolecular polymerization of $\mathrm{Gd}(\mathrm{III})$-DTPA-BTA was shown to be strongly dependent on $c_{\mathrm{NaCl}}{ }^{25}$ Fits of circular dichroism (CD) cooling curves with a nucleation-elongation model for self-assembly showed that the polymerization mechanism is nearly isodesmic $\left(K_{\mathrm{a}}=0.1\right)$ at $0.10 \mathrm{M} \mathrm{NaCl}$, while it is highly cooperative $\left(K_{\mathrm{a}}=4.6 \times 10^{-4}\right)$ at $0.83 \mathrm{M} \mathrm{NaCl} .^{38}$ Interestingly, a sharp increase in the cooperativity, i.e. a decrease of $K_{\mathrm{a}}$, was found between 0 and $0.2 \mathrm{M} \mathrm{NaCl}$ (Fig. 6). This nearly coincides with the strong decrease of $I_{\mathrm{sp}} /\left(I_{\mathrm{sp}}+I_{\mathrm{cyl}}\right)$ as obtained by SAXS, indicating a critical ionic strength at and above which long cylindrical aggregates are formed. To the best of our knowledge this is the first time a cooperative polymerization mechanism was experimentally shown to give a bimodal distribution of small oligomers and long one-dimensional helical aggregates. The appearance of a bimodal distribution of species is characteristic of cooperative self-assembly; therefore we conclude that the morphological transition observed with SAXS and cryo-TEM is driven by a salt induced increase in the cooperativity of the polymerization.

Finally we note that a sphere-to-cylinder transition is well known for block-copolymer and surfactant micelles. This transition is extensively studied as a function of concentration and/ or ionic strength for several systems. ${ }^{39,40}$ In these systems cylinders are only formed if the Israelachvili packing parameter ${ }^{41}$ 'encodes' for the formation of cylindrical micelles, provided that translational entropy is sufficiently low to be

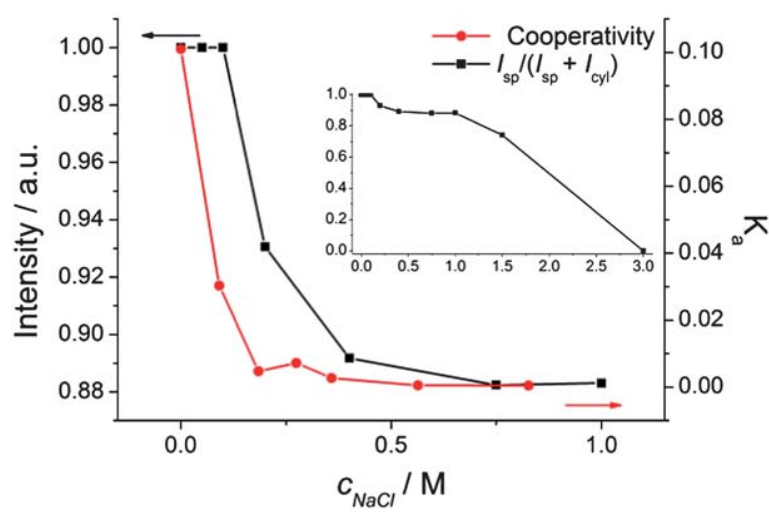

Fig. 6 Comparison of the cooperativity (right axis) of the polymerization mechanism of $\mathrm{Gd}\left(\right.$ (II)-DTPA-BTA at different $\mathrm{NaCl}$ concentrations (circles), ${ }^{25}$ with the fraction of spherical particles of the total (left axis), as determined by comparing the relative contribution to scattering intensity $I,(q \rightarrow 0)$ (squares). The inset depicts the full range of acquired SAXS data while the main graph exclusively shows the range where data for both measurements were acquired to emphasize the resemblance of structural and mechanistic transitions. 
overcome. The transition can then be triggered by an increase of the amphiphile concentration, or by a significant modification of the packing parameter in response to changes in the ionic strength, $\mathrm{pH}$ or solvent composition. The packing parameter of Gd(III)-DTPA-BTAs, however, always encodes for cylindrical objects by means of the triple hydrogen bonding motif of the BTAs. ${ }^{42}$ At low ionic strength long cylindrical objects are not observed because growth into long objects is inhibited by the electrosteric crowding of the charged ligands; an increase in $c_{\mathrm{NaCl}}$ alleviates this impediment and long rods are formed.

\section{Conclusions}

In this study we performed fluorescence, SAXS and cryo-TEM experiments to gain insight into the morphological transition from small objects at low ionic strength to long supramolecular polymers at high ionic strength. We related the morphology of the aggregates at various $\mathrm{NaCl}$ concentrations to the cooperativity of the polymerization mechanism as obtained by thermodynamic modeling of experimental CD spectroscopy data. At low $\mathrm{NaCl}$ concentrations, corresponding to a nearly isodesmic growth, we observed a monomodal distribution of low aspect ratio aggregates, whereas a bimodal distribution of small and long one-dimensional helical aggregates was observed at high $\mathrm{NaCl}$ concentrations. Furthermore we showed that there is a critical salt concentration at, and above, which long polymers begin to grow. At this same concentration the polymerization mechanism was shown to change from nearly isodesmic to cooperative. This gives us a handle to control the shape and size of the aggregates and will ultimately allow us to modify the pharmacokinetic, and contrast enhancing properties of $\mathrm{Gd}(\mathrm{III})$ DTPA-BTAs.

\section{Acknowledgements}

We thank Paul H. H. Bomans for the cryoTEM imaging. The small angle X-ray experiments were performed at the European Synchrotron Radiation Facility (ESRF) in Grenoble at the DUBBLE beamline, BM26. We are grateful for the beam time and we kindly acknowledge Giuseppe Portale for technical support. We acknowledge the Netherlands Organisation for Scientific Research (NWO VENI Grant 700.10.406) [I.K.V.], the Dutch Ministry of Education, Culture and Science (Gravity program 024.001.035), and the European Research Council (FP7/20072013, ERC Grant Agreement 246829) [E.W.M].

\section{Notes and references}

1 J. M. Lehn, Chem. Soc. Rev., 2007, 36, 151.

2 L. Brunsveld, B. J. B. Folmer, E. W. Meijer and R. P. Sijbesma, Chem. Rev., 2001, 101, 4071.

3 S. Cantekin, T. F. A. de Greef and A. R. A. Palmans, Chem. Soc. Rev., 2012, 41, 6125.

4 S. I. Stupp, V. LeBonheur, K. Walker, L. S. Li, K. E. Huggins, M. Keser and A. Amstutz, Science, 1997, 276, 384.

5 P. Y. W. Dankers, T. M. Hermans, T. W. Baughman, Y. Kamikawa, R. E. Kieltyka, M. M. C. Bastings,
H. M. Janssen, N. A. M. J. Sommerdijk, A. Larsen, M. J. A. van Luyn, A. W. Bosman, E. R. Popa, G. Fytas and E. W. Meijer, Adv. Mater., 2012, 24, 2703.

6 J. Boekhoven, J. M. Poolman, C. Maity, F. Li, L. van der Mee, C. B. Minkenberg, E. Mendes, J. H. van Esch and R. Eelkema, Nat. Chem., 2013, 5, 433.

7 R. E. Kieltyka, A. C. H. Pape, L. Albertazzi, Y. Nakano, M. M. C. Bastings, I. K. Voets, P. Y. W. Dankers and E. W. Meijer, J. Am. Chem. Soc., 2013, 135, 11159.

8 L. E. O'Leary, J. A. Fallas, E. L. Bakota, M. K. Kang and J. D. Hartgerink, Nat. Chem., 2011, 3, 821.

9 K. Petkau-Milroy, M. H. Sonntag, A. H. A. M. van Onzen and L. Brunsveld, J. Am. Chem. Soc., 2012, 134, 8086.

10 P. Besenius, J. L. M. Heynens, R. Straathof, M. M. L. Nieuwenhuizen, P. H. H. Bomans, E. Terreno, S. Aime, G. J. Strijkers, K. Nicolay and E. W. Meijer, Contrast Media Mol. Imaging, 2012, 7, 356.

11 S. R. Bull, M. O. Guler, R. E. Bras, T. J. Meade and S. I. Stupp, Nano Lett., 2004, 5, 1-4.

12 B. C. Gibb, Isr. J. Chem., 2011, 51, 798.

13 E. Obert, M. Bellot, L. Bouteiller, F. Andrioletti, C. LehenFerrenbach and F. Boué, J. Am. Chem. Soc., 2007, 129, 15601.

14 G. M. Pawar, M. Koenigs, Z. Fahimi, M. Cox, I. K. Voets,

H. M. Wyss and R. P. Sijbesma, Biomacromolecules, 2012, 13, 3966.

15 D. Görl, X. Zhang and F. Würthner, Angew. Chem., Int. Edit., 2012, 51, 6328.

16 A. R. M. Soares, J. P. C. Tomé, M. G. P. M. S. Neves, A. C. Tomé, J. A. S. Cavaleiro and T. Torres, Carbohyd. Res., 2009, 344, 507.

17 Y.-b. Lim, K.-S. Moon and M. Lee, J. Mater. Chem., 2008, 18, 2909.

18 S.-i. Tamaru, M. Nakamura, M. Takeuchi and S. Shinkai, Org. Lett., 2001, 3, 3631.

19 G. J. Richards, J. Labuta, J. P. Hill, T. Mori and K. Ariga, J. Phys. Chem. Lett., 2010, 1, 1336.

20 P. J. M. Stals, M. M. J. Smulders, R. Martín-Rapún, A. R. A. Palmans and E. W. Meijer, Chem.-Eur. J., 2009, 15, 2071.

21 C. M. A. Leenders, L. Albertazzi, T. Mes, M. M. E. Koenigs, A. R. A. Palmans and E. W. Meijer, Chem. Commun., 2013, 49, 1963.

22 L. Albertazzi, F. J. Martinez-Veracoechea, C. M. A. Leenders, I. K. Voets, D. Frenkel and E. W. Meijer, Proc. Natl. Acad. Sci. U. S. A., 2013, 110, 12203.

23 P. Besenius, Y. Goedegebure, M. Driesse, M. Koay, P. H. H. Bomans, A. R. A. Palmans, P. Y. W. Dankers and E. W. Meijer, Soft Matter, 2011, 7, 7980.

24 M. von Gröning, I. de Feijter, M. C. A. Stuart, I. K. Voets and P. Besenius, J. Mater. Chem. B, 2013, 1, 2008.

25 P. Besenius, G. Portale, P. H. H. Bomans, H. M. Janssen, A. R. A. Palmans and E. W. Meijer, Proc. Natl. Acad. Sci. U. S. A., 2010, 107, 17888.

26 C. Schaefer, I. K. Voets, A. R. A. Palmans, E. W. Meijer, P. van der Schoot and P. Besenius, ACS Macro Lett., 2012, 1, 830.

27 A. Bernheim-Groswasser, R. Zana and Y. Talmon, J. Phys. Chem. B, 2000, 104, 4005. 
28 M. Nakano, H. Matsuoka, H. Yamaoka, A. Poppe and D. Richter, Macromolecules, 1999, 32, 697.

29 P. R. Majhi, P. L. Dubin, X. Feng, X. H. Guo, F. A. M. Leermakers and C. Tribet, J. Phys. Chem. B, 2004, 108, 5980.

30 M. Pisárčik, M. Dubničková, F. Devínsky, I. Lacko and J. Škvarla, Colloids Surf., A, 1998, 143, 69.

31 M. Sutter, S. Oliveira, N. N. Sanders, B. Lucas, A. Hoek, M. A. Hink, J. W. G. Visser, S. C. de Smedt, W. E. Hennink and W. Jiskoot, J. Fluoresc., 2007, 17, 181.

32 R. Mishra, D. Sjölander and P. Hammarström, Mol. BioSyst., 2011, 7, 1232-1240.

33 B. Demeule, R. Gurny and T. Arvinte, Int. J. Pharm., 2007, 329, 37.

34 A. Hawe, M. Sutter and W. Jiskoot, Pharm. Res., 2008, 25, 1487.
35 J. Boekhoven, A. M. Brizard, P. van Rijn, M. C. A. Stuart, R. Eelkema and J. H. van Esch, Angew. Chem., Int. Ed., 2011, 50, 12285.

36 P. Greenspan and S. D. Fowler, J. Lipid Res., 1985, 26, 781.

37 M. C. A. Stuart, J. C. van de Pas and J. B. F. N. Engberts, J. Phys. Org. Chem., 2005, 18, 929.

38 P. van der Schoot, in Supramolecular Polymers, ed. A. Ciferri, Taylor \& Francis, 2nd edn, 2005.

39 H. M. van der Kooij, E. Spruijt, I. K. Voets, R. Fokkink, M. A. C. Stuart and J. van der Gucht, Langmuir, 2012, 28, 14180.

40 R. Lund, V. Pipich, L. Willner, A. Radulescu, J. Colmenero and D. Richter, Soft Matter, 2011, 7, 1491.

41 J. N. Israelachvili, D. J. Mitchell and B. W. Ninham, J. Chem. Soc., Faraday Trans. 2, 1976, 72, 1525.

42 M. P. Lightfoot, F. S. Mair, R. G. Pritchard and J. E. Warren, Chem. Commun., 1999, 19, 1945. 